\title{
Anomalous surface-enhanced Raman scattering of aromatic aldehydes and carboxylic acids
}

\author{
N. F. L. Machado, ${ }^{a, b}$ M. P. M. Marques, ${ }^{b, c}$ L. A. E. Batista de Carvalho, ${ }^{b}$ \\ J. L. Castro ${ }^{\mathrm{a}}$ and J. C. Otero ${ }^{\mathrm{a} *}$
}

Raman and SERS spectra of benzaldehyde (Bz-CHO) and chromone-3-carboxaldehyde (Ch-CHO) on silver colloids have been analyzed, being subsequently compared to the spectra of the corresponding acids. In the SERS spectra of both aldehydes, the band corresponding to the $v(C=0)$ stretching mode of the carboxaldehyde group at $c a .1700 \mathrm{~cm}^{-1}$ is missing, while a new band at $1350-$ $1400 \mathrm{~cm}^{-1}$, characteristic of the symmetric stretching mode of carboxylate group, is appearing in both cases. These results point out that aldehydes are oxidized to their corresponding acids when adsorbed on silver nanoparticles. This conclusion has been confirmed by means of HPLC-MS analysis and supported on the basis of DFT calculations. Copyright $\odot 2016$ John Wiley \& Sons, Ltd. Additional supporting information may be found in the online version of this article at the publisher's web site.

Keywords: SERS; aldehyde; carboxylic acid; oxidation; nanoparticles

\section{Introduction}

Surface-enhanced raman scattering (SERS) is a complex phenomenon characterized by the huge enhancement of the Raman signal of molecules adsorbed on nanostructured surfaces of some metals, mainly $\mathrm{Ag}, \mathrm{Cu}$ and $\mathrm{Au}$. The high sensitivity of this technique allows to retrieve detailed information concerning the adsorbed species at very low concentrations, such as the functional groups that are in contact with the metal surface or the conformation of the adsorbates. But the interpretation of each particular SERS can be a challenge, given that the relative intensities and wavenumbers of the Raman and the corresponding SERS spectra can be very different because of the involvement of different enhancement mechanisms and the adsorption process on the metal surface. Concerning the differences between the Raman and SERS relative intensities, different surface selection rules have been proposed in order to account for the selective enhancement of specific SERS bands. The most popular rules were derived from the so-called electromagnetic (EM) enhancement mechanism of SERS (EM-SERS), being focused on comparing the intensification or weakening of specific SERS bands depending on the surface orientation of the adsorbate. More complex selection rules are derived from the additional enhancement mechanism related to resonance Raman effects involving excited electronic states of the molecule (surface-enhanced resonant Raman scattering, SERRS), metal-adsorbate photoinduced charge transfer (CT) processes (CT-SERS) or another electronic resonances of the surface complex. ${ }^{[1,2]}$ The problem is to known which SERS mechanism or mechanisms are acting in each particular experiment, but this basic and key question remains controversial 40 years after the discovery of the phenomenon.

Furthermore, some SERS bands can be originated by chemical species different from the original sample. Impurities or new species produced by chemical transformations induced by the laser excitation and/or catalyzed by the metal surface can dominate the spectra. ${ }^{[3]}$ The aromatic aldehydes are a good example of this given that their SERS show very often marked differences with respect to the Raman records. These discrepancies have been explained, for instance, by supposing the formation of the respective alcohols, among other products, ${ }^{[4-6]}$ but the reverse reaction is also proposed in other cases as the benzyl alcohol, which seems to be oxidized to benzaldehyde. ${ }^{[7]}$ Alternatively, oxidation of aromatic ${ }^{[8]}$ or aliphatic aldehydes ${ }^{[9]}$ has been detected in SERS giving the spectra of the corresponding carboxylic derivatives.

We have shown that the occurrence of resonant $\mathrm{CT}$ processes (SERS-CT) in the SERS of benzene and others aromatic derivatives is characterized by the selective enhancement of the very characteristic ring stretching band corresponding to the Wilson's $\phi 8 \mathrm{a}$ mode ${ }^{[10]}$ recorded at $c a .1600 \mathrm{~cm}^{-1} \cdot{ }^{[11-13]}$ This is the case of the SERS of chromone-3-carboxylic acid (Ch- $\mathrm{COOH})$, which adsorbs on the surface in its anionic form, ${ }^{[14]}$ whereas the SERS of chromone3-carboxaldehyde (Ch-CHO) here reported displays an anomalous behavior given that looks like the corresponding spectrum of the acid. The present work aims to account for these results, which are explained by the occurrence of a chemical oxidation of the aldehyde catalyzed by the silver surface. In order to support this conclusion, the Raman and SERS of both chromones, $\mathrm{Ch}-\mathrm{CHO}$ and $\mathrm{Ch}-$ $\mathrm{COOH}$, have been compared with the corresponding spectra of benzaldehyde $(\mathrm{Bz}-\mathrm{CHO})$ and benzoic acid $(\mathrm{Bz}-\mathrm{COOH})$ finding that the spectra of the two pairs of derivatives show a similar behavior.

* Correspondence to: Juan C. Otero, Universidad de Málaga, Andalucía Tech, Facultad de Ciencias, Departamento de Química Física, Unidad Asociada CSIC, 29071 Málaga, Spain.

E-mail:jc_otero@uma.es

a Andalucía Tech, Facultad de Ciencias, Departamento de Química Física, Unidad Asociada CSIC, Universidad de Málaga, 29071, Málaga, Spain

b Química-Física Molecular, Departamento de Química FCTUC, Universidade de Coimbra, 3004-535, Coimbra, Portugal

c Departamento Ciências da Vida FCTUC, Universidade de Coimbra, 3000-456, Coimbra, Portugal 


\section{Experimental}

\section{Samples and instrumentation}

Colloidal silver sols have been prepared in deionized and triply distilled water according to the method described by Creighton et al. ${ }^{[15]}$ One volume of $10^{-3} \mathrm{M} \mathrm{AgNO}_{3}$ is added dropwise to three volumes of $2 \times 10^{-3} \mathrm{M} \mathrm{NaBH}_{4}$, which have been previously cooled to a temperature between 0 and $5^{\circ} \mathrm{C}$. After stirring the mixture for a short period till homogenization, it is allowed to rest at room temperature for approximately $90 \mathrm{~min}$. The result is a silver colloidal solution displaying yellow color, with a maximum in its absorbance spectrum at $390 \mathrm{~nm}$, which remains stable for several months. Adsorbate is then added to the colloid as an aqueous solution up to obtain the desired concentration. A change in the color of the system, from the initial yellowish to a final blue-greenish, is observed because of nanoparticle aggregation when the adsorbate molecule is added to the colloid and surface adsorption occurs. The aggregation is the cause of the appearance of new very broad absorption bands in the VIS spectrum which are red-shifted with respect to the silver plasmon at $390 \mathrm{~nm}$.

Raman spectra were recorded with a Jobin-Yvon U-1000 double monocromator spectrometer fitted with a cooled Hamamatsu R943-02 photomultiplier, using the 514.5-nm exciting line from a Spectra Physics $2020 \mathrm{Ar}^{+}$gas laser. A constant slit width was used allowing a spectral resolution of $4 \mathrm{~cm}^{-1}$. The laser power reaching the sample was always $60 \mathrm{~mW}$. In the case of liquid samples a quartz cell of $1.0-\mathrm{cm}$ path length was used, while a glass capillary was used for the microcrystalline solids. The measurement of the wavenumbers was undertaken resorting to the laser plasma lines as standards, whereby an accuracy of $\pm 1 \mathrm{~cm}^{-1}$ was obtained under the operating conditions employed.

High-performance liquid chromatography-mass spectrometry (HPLC-MS) spectra were recorded with an Accela ${ }^{T M}$ High Speed LC system, coupled to a TSQ Quantum Access MAX Triple Stage Quadrupole Mass Spectrometer (Thermo Electron Corporation).

\section{DFT calculations}

Quantum mechanical calculations were carried out with the GAUSSIAN 03 W software package ${ }^{[16]}$ within the density functional theory (DFT) approach. The widely employed B3LYP hybrid method, which includes a mixture of Hartree-Fock (HF) and DFT exchange terms and the gradient-corrected correlation functional of Lee, Yang and Parr ${ }^{[17,18]}$ as proposed and parameterised by Becke, ${ }^{[19,20]}$ was used along with the effective core potential LanL2DZ basis set. ${ }^{[21]}$ Molecular geometries without geometrical constraints were fully optimized. Harmonic force field has been calculated at the same theory level used for the geometry optimisation.

\section{Results and discussion}

\section{Raman and SERS spectra of chromone-3-carboxaldehyde (Ch-CHO)}

The Raman of solid as well as the SERS spectra on silver sols at a concentration of $10^{-3} \mathrm{M}$ and $\mathrm{pH}=7$ of $\mathrm{Ch}-\mathrm{CHO}$ and $\mathrm{Ch}-\mathrm{COOH}$ (chromone-3-carboxylic acid) are displayed in Fig. 1, and the assignments of the recorded bands are summarized in Table S1 (Supporting Information). The Raman spectra of these two species show important differences, mainly in the spectral region around

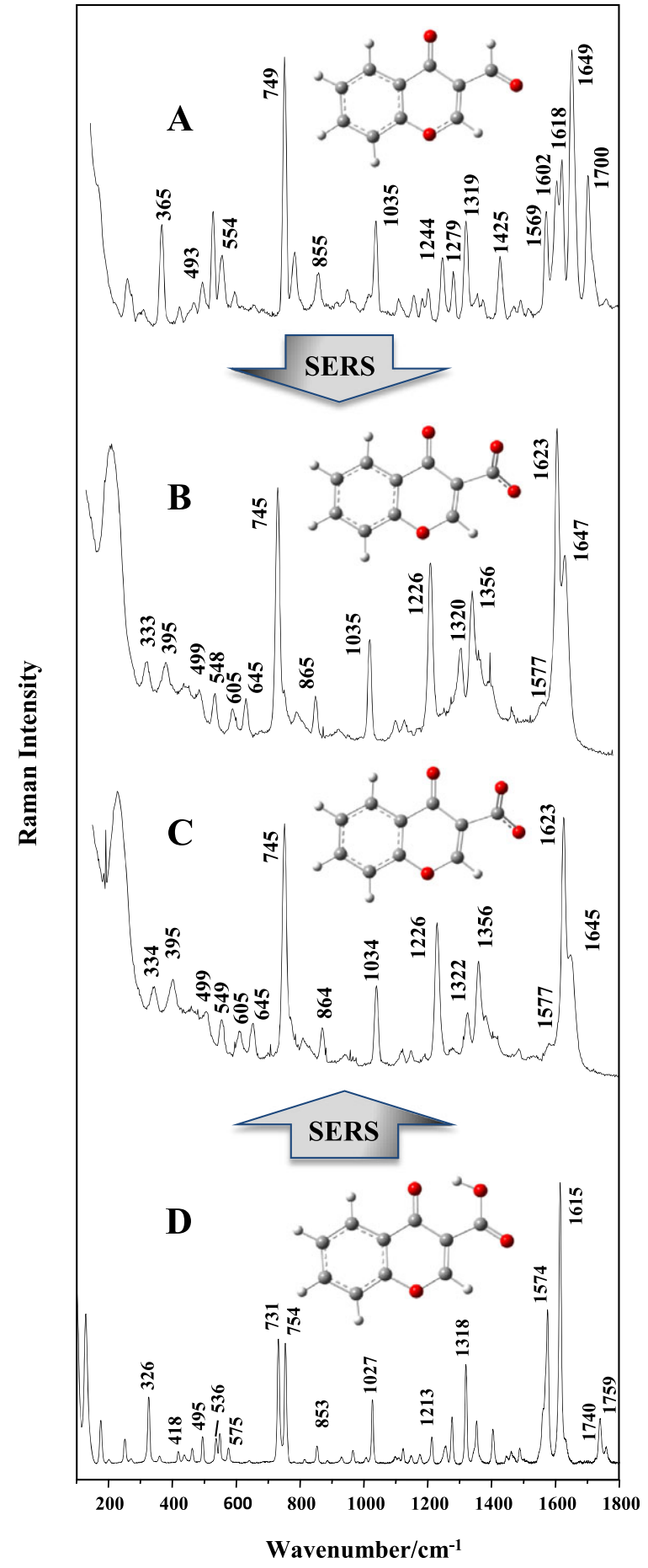

Figure 1. Raman spectrum of solid (A) and SERS (B) of Ch-CHO. SERS (C) and Raman spectrum of solid (D) $\mathrm{Ch}-\mathrm{COOH}$. The molecular models correspond to the chemical species responsible for the observed bands. [Colour figure can be viewed at wileyonlinelibrary.com]

$1600 \mathrm{~cm}^{-1}$ (Fig. 1, A and D). Two strong bands are found at 1574 and $1615 \mathrm{~cm}^{-1}$ in the case of the acid which, according to previous work, ${ }^{[14]}$ have been assigned to the $\phi 8 \mathrm{~b}$ and $\phi 8 \mathrm{a}$ Wilson's ringstretching modes, respectively. Besides, a weak band at $1740 \mathrm{~cm}^{-1}$ is also seen, being assigned to the $v(C=O)_{\text {carbox }}$. fundamental. ${ }^{[14]}$

Concerning the Raman spectra of the corresponding aldehyde, a strong band at $1649 \mathrm{~cm}^{-1}$ as well as four medium bands are recorded 
in the same region at $1569,1602,1618$ and $1700 \mathrm{~cm}^{-1}$, in agreement with the predicted wavenumbers from the calculated force field (Fig. 1A, Tables S1 and S2 of Supporting Information). The strongest band can be assigned to a vibrational mode involving $v(C=O)_{\text {ald }}$ and $v(C=O)$ pyrone coordinates coupled with the $\phi 8$ a motion, while the band at $1700 \mathrm{~cm}^{-1}$ is mainly originated by the $v(C=O)_{\text {ald }}$ stretching. The bands at 1618 and $1569 \mathrm{~cm}^{-1}$ can be assigned to the $\phi 8 \mathrm{a}$ and $\phi 8 \mathrm{~b}$ modes, respectively. The last fundamental is coupled to $v(C=C)$ stretching, while the $1602 \mathrm{~cm}^{-1}$ line contains a more complex mixing of $\phi 8 \mathrm{a}, \phi 8 \mathrm{~b}$ and $v(\mathrm{C}=\mathrm{C})$ contributions.

By comparing the normal Raman and SERS spectra of $\mathrm{Ch}-\mathrm{CHO}$ (Fig. 1, A and B), it can be seen that the lines at 1425 and $1700 \mathrm{~cm}^{-1}$ are missing in SERS. According to the force field calculations, these bands correspond to vibrational modes related to the aldehyde group (Table S1 of Supporting Information), as is also the case of the band at $1602 \mathrm{~cm}^{-1}$ which is not observed in the SERS spectrum of Ch-CHO (Fig. 1B, Table S1 of Supporting Information). On the contrary, a new band is observed in SERS, but not in normal Raman, with medium intensity at $1356 \mathrm{~cm}^{-1}$, corresponding to the characteristic wavenumber of the $v_{s}(O C O)$ mode of the carboxylate group.

These results point out that $\mathrm{Ch}-\mathrm{CHO}$ might not be the chemical species which originates the SERS shown in Fig. 1B. The SERS of $\mathrm{Ch}-\mathrm{CHO}$ and $\mathrm{Ch}-\mathrm{COOH}$ (Fig. $\mathrm{B}$ and $1 \mathrm{C}$ ) show very similar relative intensities and vibrational wavenumbers and, moreover, are characterized by the appearance of the symmetric stretching of the carboxylate group at $1356 \mathrm{~cm}^{-1}$ given that the organic acids are adsorbed ionized $\left(\mathrm{Ch}-\mathrm{COO}^{-}\right)$on the metal surface. ${ }^{[14]}$ In this respect, the strongest bands recorded in the SERS of the acid at $745,1226,1356,1623$ and $1645 \mathrm{~cm}^{-1}$ are recorded with similar intensities in the SERS of the aldehyde and have been assigned as $\delta(\mathrm{OCO})+\phi 6, \phi 9 \mathrm{a}, v_{\mathrm{s}}(\mathrm{OCO}), \phi 8 \mathrm{a}$ and $\mathrm{v}(\mathrm{C}=\mathrm{O})$, respectively (Table S1 of Supporting Information).

Table S2 (Supporting Information) summarizes the assignment of the most characteristic vibrational modes calculated for the $\mathrm{Ch}-\mathrm{CHO}, \mathrm{Ch}-\mathrm{COOH}$ and $\mathrm{Ch}-\mathrm{COO}^{-}$species as well as the corresponding wavenumbers calculated for the $\mathrm{Ch}-\mathrm{CHO} / \mathrm{Ag}^{+}$and $\mathrm{Ch}_{-} \mathrm{COO}^{-}$/ $\mathrm{Ag}^{+}$complexes. Concerning the aldehyde, the Raman bands recorded at 1618,1649 and $1700 \mathrm{~cm}^{-1}$ correlate with the calculated at 1645,1664 and $1682 \mathrm{~cm}^{-1}$, respectively, while the first two are predicted at 1660 and $1662 \mathrm{~cm}^{-1}$ in the case of $\mathrm{Ch}-\mathrm{CHO} / \mathrm{Ag}^{+} \mathrm{com}-$ plex. The band at higher wavenumber $\left(1700 \mathrm{~cm}^{-1}\right)$ has no direct counterpart in the force field of the complex because the $v(C=O)$ ald mode is red-shifted because of the mixing with other coordinates when the aldehyde is strongly bonded to the silver cation. Even though this could explain the disappearance of the latter band in the SERS spectrum of the aldehyde, the absence in SERS of the bands recorded in normal Raman at 1425 and $1602 \mathrm{~cm}^{-1}$ and the appearance in SERS of the $1356 \mathrm{~cm}^{-1}$ line cannot be justified on the basis of the theoretical calculations carried out for the aldehyde bonded to $\mathrm{Ag}^{+}$.

Therefore, it seems evident that the $\mathrm{Ch}-\mathrm{CHO}$ undergoes a chemical transformation in the SERS conditions, being oxidized and adsorbed, as usual, on the positively charged nanoparticles as carboxylate anion. For instance, we have reported that aliphatic aldehydes are oxidized in silver sols, giving rise to their corresponding carboxylic acids ${ }^{[9]}$ and a similar transformation has been detected in the SERS of 4-dimethylaminobenzaldehyde recorded on gold nanoparticles. ${ }^{[8]}$ The chemical reaction can be catalyzed by the heterogeneous surface of silver and the oxidating agent could be the atmospheric oxygen dissolved in the sol or the oxidized shell of the nanoparticles. ${ }^{[22,23]}$

\section{Raman and SERS spectra of benzaldehyde (Bz-CHO)}

Figure 2 shows the Raman spectra of pure liquid benzaldehyde (Bz-CHO) and solid benzoic acid $(\mathrm{Bz}-\mathrm{COOH})$ as well as the respective SERS recorded on silver sols at $10^{-3} \mathrm{M}$ and $\mathrm{pH}=7$. The assignments of these spectra are summarized in Table S3 (Supporting Information). Significant differences can be observed when the respective Raman spectra are compared (Fig. 2, A and D). The spectrum of

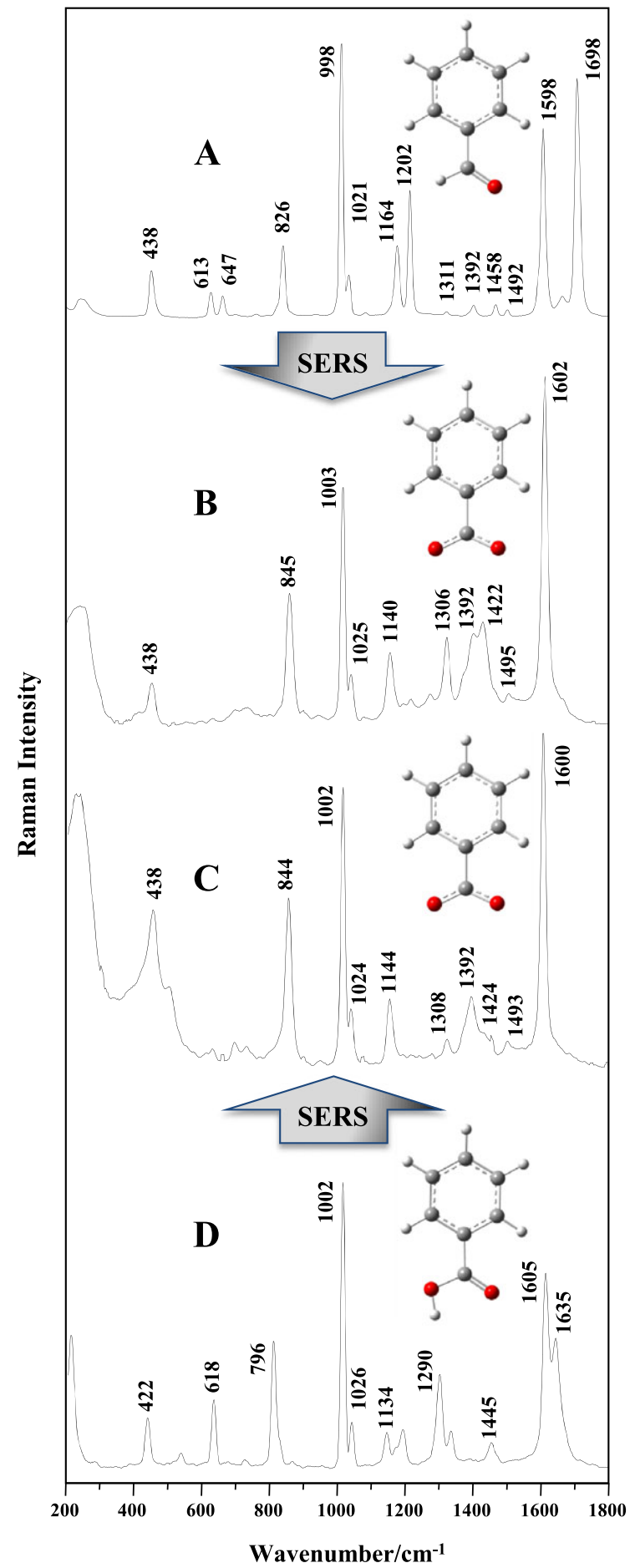

Figure 2. Raman spectrum of pure liquid (A) and SERS (B) of Bz-CHO. SERS (C) and Raman spectrum of solid (D) $\mathrm{Bz}-\mathrm{COOH}$. The molecular models correspond to the chemical species responsible for the observed bands. [Colour figure can be viewed at wileyonlinelibrary.com] 
the acid is characterized by the bands at 1290 and $1635 \mathrm{~cm}^{-1}$ related to vibrational modes of the carboxylic group. Similarly, the - $\mathrm{CHO}$ group of the aldehyde derivative is responsible for the strong Raman lines recorded at 1202 and $1698 \mathrm{~cm}^{-1}$, respectively (Table S3 of Supporting Information). Both compounds are monosubstituted benzenes and show the characteristic vibrations of this kind of derivatives. However, some aromatic ring modes display differentiated wavenumbers in each molecule. For instance, the breathing mode $\phi 1$ of the acid is recorded at $796 \mathrm{~cm}^{-1}$ in the Raman spectrum given that is strongly coupled with the carboxylic group bending, whereas it is observed at $826 \mathrm{~cm}^{-1}$ in the spectrum of $\mathrm{Bz}-\mathrm{CHO}$. Likewise, the Raman band at $422 \mathrm{~cm}^{-1}$ of $\mathrm{Bz}-\mathrm{COOH}$, assigned as an in-plane ring deformation, is recorded at $438 \mathrm{~cm}^{-1}$ in the spectrum of the aldehyde. The mentioned differences are reproduced by the B3LYP/LanL2DZ force field (Table S4 of Supporting Information). Table S3 (Supporting Information) also summarizes the Raman assignment of the fundamentals of benzoate anion observed in the spectrum of the $1 \mathrm{M}$ aqueous solution of benzoic acid in basic medium $(\mathrm{pH}=14)$. The main differences with the spectrum of the solid are the new band recorded at $1390 \mathrm{~cm}^{-1}$ in the solution, corresponding to $v_{s}(\mathrm{OCO})$ of the ionized carboxylate group, and the blue-shift of $\phi 1$ mode $\left(+46 \mathrm{~cm}^{-1}\right)$, being both results reproduced by the DFT calculations.

The normal Raman and SERS spectra of Bz-CHO show noticeable differences, namely the absence in SERS of the strong bands at 1698 and $1202 \mathrm{~cm}^{-1}$ related to the $-\mathrm{CHO}$ group (Tables S3 and S4 of Supporting Information), the appearance of a new SERS band at $1392 \mathrm{~cm}^{-1}$ and the significant wavenumber shifts show by several bands (Fig. 2, A and B).

The SERS spectrum of benzoic acid (Fig. 2C) has been the subject of several previous studies. ${ }^{[1,24-28]}$ This spectrum differs from the Raman of the solid acid (Fig. 2D) while correlates well with the Raman of solutions at high $\mathrm{pH}$ where the acid is ionized. The Raman bands at 1635 and $1290 \mathrm{~cm}^{-1}$, characteristic of the $-\mathrm{COOH}$ group, are missing in the SERS and a new band is recorded with medium intensity at $1392 \mathrm{~cm}^{-1}$ corresponding to the symmetric stretching mode of the carboxylate group, $v_{s}(\mathrm{OCO})$, originated when the acid deprotonates upon adsorption on the metal surface (Table S3 of Supporting Information). On the other hand, the selective enhancement observed in SERS of the stretching ring mode $\phi 8 a$ at $1600 \mathrm{~cm}^{-1}$ with respect to either the pure solid acid or the aqueous solution at $\mathrm{pH}=14$ Raman spectra, is because of a resonant CT process as discussed elsewhere. ${ }^{[1]}$

It can be appreciated that the SERS of $\mathrm{Bz}-\mathrm{CHO}$ and $\mathrm{Bz}-\mathrm{COOH}$ shown in Fig. $2 \mathrm{~B}$ and $\mathrm{C}$ are remarkably similar. The majority of the main SERS bands are common to both spectra, with wavenumber differences smaller than $4 \mathrm{~cm}^{-1}$. Regarding the intensity of the bands, only minor differences can be noticed: the band recorded at $1422 \mathrm{~cm}^{-1}$ in the SERS of the aldehyde has stronger intensity than in the spectrum of the acid, while the band at $438 \mathrm{~cm}^{-1}$ is more intense in the case of the acid than in the SERS of the aldehyde. These minor changes may be because of the intrinsic properties of the colloids, which can produce slight differences in the SERS intensities, even for the same molecule, when different samples of sols are used or are recorded at different times.

The optimized geometries and the vibrational wavenumbers of selected molecular systems calculated at the B3LYP/LanL2DZ level of theory are shown in Table S4 (Supporting Information). This table summarizes a selected set of wavenumbers calculated for $\mathrm{Bz}-\mathrm{CHO}$ and $\mathrm{Bz}-\mathrm{COOH}$ (monomer and its centrosymmetric dimer), $\mathrm{Bz}^{-} \mathrm{COO}^{-}$ and $\mathrm{Bz}-\mathrm{COO}^{-} \mathrm{Na}^{+}(\mathrm{aq})$ (including two water molecules in the model), and the respective silver complexes $\mathrm{Bz}-\mathrm{CHO} / \mathrm{Ag}^{+}$and $\mathrm{Bz}-\mathrm{COO}^{-} / \mathrm{Ag}^{+}$ complexes. The models of $\mathrm{Bz}_{-} \mathrm{COO}^{-} \mathrm{Na}^{+}(\mathrm{aq})$ and the $\mathrm{Bz}-\mathrm{COOH}$ dimer have been selected according to ref. 29 and are drawn in Fig. S1 (Supporting Information), where the intermolecular hydrogen bond distances are detailed. The Raman spectrum of solid benzoic correlates very well with the calculated force field of the dimer which predicts the most characteristic bands of the acid at 1635, 1290, 1002 and $796 \mathrm{~cm}^{-1}$ in agreement with the experimental results (Table S4 of Supporting Information). For instance, the bands recorded at 1290 and $796 \mathrm{~cm}^{-1}$ are calculated at 1321 and $792 \mathrm{~cm}^{-1}$ and are assigned to $\mathrm{OH}$ in-plane bending and the ring mode $\phi 1$, respectively. The last one is strongly coupled with the $v(C=O)$ stretching and OCO in-plane bending. It must be stressed that the wavenumbers of $v_{s}(\mathrm{OCO})$ and $\phi 1$ vibrational modes of $\mathrm{Bz}-\mathrm{COO}^{-}$are calculated at 1289 and $789 \mathrm{~cm}^{-1}$, respectively, for the isolated benzoate and at 1401 and $820 \mathrm{~cm}^{-1}$ by assuming the $\mathrm{Bz}_{-} \mathrm{COO}^{-} \mathrm{Na}^{+}(\mathrm{aq})$ model, giving in this case a better agreement with the experimental values of 1390 and $842 \mathrm{~cm}^{-1}$, respectively (Table S3 of Supporting Information).

In the case of the aldehyde, the calculated vibrational wavenumbers for the isolated molecule (Table S4 of Supporting Information) agree with the results recorded in the Raman spectrum (Fig. 2A), while no good correlation is found when the results calculated for the $\mathrm{Bz}-\mathrm{CHO} / \mathrm{Ag}^{+}$complex are compared with the SERS spectrum shown in Fig. 2B. For instance, according to the theoretical calculations the fundamental at $1202 \mathrm{~cm}^{-1}$ in Fig. $2 \mathrm{~A}$ should be shifted up to ca. $1280 \mathrm{~cm}^{-1}$ in SERS, but such behavior is not observed experimentally. Furthermore, the $\mathrm{Bz}-\mathrm{CHO} / \mathrm{Ag}^{+}$model does not justify the absence of the Raman band at $1698 \mathrm{~cm}^{-1}$ in SERS, neither the appearance of a new SERS band at $1392 \mathrm{~cm}^{-1}$. However, the calculations carried out by assuming the $\mathrm{Bz}-\mathrm{COO}^{-} / \mathrm{Ag}^{+}$complex allow for reproducing the experimental SERS obtained for both, the acid and the benzaldehyde molecules, and justify the appearance of the band at ca. $1390 \mathrm{~cm}^{-1}$ in both cases and the blue-shift of the $\phi 1$ mode of the acid from 796 to $842 \mathrm{~cm}^{-1}$. All these results point out to the oxidation of the benzaldehyde in the SERS experimental conditions, giving the formation of the corresponding carboxylic acid which is adsorbed ionized as discussed in the case of $\mathrm{Ch}-\mathrm{CHO}$.

HPLC-MS analysis of adsorbate/sol samples has been carried out for the aldehydes and the corresponding acids in order to confirm this conclusion (Fig. S2 of Supporting Information). These spectra reveal that the aldehyde group is completely oxidized to carboxylic acid in case of the $\mathrm{Bz}-\mathrm{CHO} / \mathrm{sol}$ samples. On the contrary, the peak corresponding to the acid is also detected in the $\mathrm{Ch}-\mathrm{CHO} / \mathrm{sol}$, but with very weak intensity when compared with the very strong signal because of the aldehyde itself. Nevertheless, the SERS spectrum of $\mathrm{Ch}$-CHO is completely dominated by the corresponding ionized form of the acid, what implies that the aldehyde and carboxylate functional groups have a very different affinity for the silver surface. The carbonyl group has a poor affinity for the metal what explains the difficulties for recording the SERS of organic aldehydes or ketones.

In order to know the oxidation mechanism it would be necessary to perform additional experiments, for instance, to prepare the samples in dark room and under inert atmosphere. It could be also interesting to repeat the SERS measurements by stirring the sample or by using gold sols for checking if the less active gold surface is also able to oxidize the aldehydes.

\section{Conclusions}

The differences found between the Raman and SERS spectra of $\mathrm{Bz}-\mathrm{CHO}$ and $\mathrm{Ch}-\mathrm{CHO}$ aldehydes point out to the occurrence of a 
chemical transformation upon adsorption on the silver nanoparticles. The Raman bands corresponding to the characteristic $v(C=O)$ stretching mode of the carboxaldehyde group, recorded at 1698 and $1700 \mathrm{~cm}^{-1}$ in $\mathrm{Bz}-\mathrm{CHO}$ and $\mathrm{Ch}-\mathrm{CHO}$, respectively, are completely missing in SERS. Moreover, it has been found that the SERS of both molecules show new bands at 1356 and $1392 \mathrm{~cm}^{-1}$, respectively, which are characteristic wavenumbers of the $v_{s}(O C O)$ symmetric stretching mode of the adsorbed carboxylate group. All these results point out to the oxidation of the aldehydes, giving the corresponding acids and explaining the similarity found between the SERS spectra obtained from either the aldehyde or the corresponding carboxylic acid derivative. This main conclusion has been supported by means of HPLC-MS which reveal the presence of the acid in the spectra of aldehyde/sol samples. These and other published results on carboxaldehydes suggest that this can be a general trend. The catalytic activity of heterogeneous silver nanoparticles is able to produce a total or partial oxidation of aliphatic and aromatic aldehydes, but the SERS spectra are dominated by the strong signals from the corresponding deprotonated acids.

\section{Acknowledgements}

This research has been funded by the Spanish/EU MINECO/FEDER (Project Number CTQ2015-65816-R) and the Portuguese Foundation for Science and Technology (Project Number UID/MULTI/ 00070/2013). N. F. L. Machado kindly acknowledges a post-doctoral research grant (BI/CITAB/UTAD/QUI/2015) from the Centre for the Research and Technology of Agro-Environmental and Biological Sciences, Portugal.

\section{References}

[1] R. Aroca, Surface-enhanced Vibrational Spectroscopy, John Wiley \& Sons Ltd, Chichester, 2006.

[2] J. Roman-Perez, I. Lopez-Tocon, J. L. Castro, J. F. Arenas, J. Soto, J. C. Otero, Phys. Chem. Chem. Phys. 2015, 17, 2326.

[3] J. F. Arenas, J. L. Castro, J. C. Otero, J. I. Marcos, J. Raman Spectrosc. 1992, 23, 249.

[4] M. R. Andersont, D. H. Evans, J. Am. Chem. Soc. 1998, 110, 6612.

[5] K. A. Bunding, M. I. Bell, Surf. Sci. 1983, 118, 329.

[6] R. L. Birke, C. Shi, W. Zhang, J. R. Lombardi, J. Phys. Chem. B 1998, 102, 7983.

[7] D. Sun, G. Zhang, X. Jiang, J. Huang, X. Jing, Y. Zheng, J. He, Q. Li, J. Mater. Chem. A 2014, 2, 1767.
[8] R. M. Krieger, P. W. Jagodzinski, J. Mol. Struct. 2008, 876, 64.

[9] J. L. Castro, J. C. Otero, J. I. Marcos, J. Raman Spectrosc. 1997, 28, 765.

[10] E. B. Wilson Jr., Phys. Rev. 1934, 45, 706.

[11] M. R. Lopez-Ramirez, C. Ruano, J. L. Castro, J. F. Arenas, J. Soto, J. C. Otero, J. Phys. Chem. C 2010, 114, 7666.

[12] S. P. Centeno, I. Lopez-Tocon, J. Arenas, J. Soto, J. C. Otero, J. Phys. Chem. B 2006, 110, 14916.

[13] J. Arenas, I. Lopez-Tocon, J. L. Castro, S. P. Centeno, M. R. Lopez-Ramirez, J. C. Otero, J. Raman Spectrosc. 2005, 36, 515.

[14] N. F. L. Machado, C. Ruano, J. L. Castro, M. P. M. Marques, J. C. Otero, Phys. Chem. Chem. Phys. 2011, 13, 1012.

[15] J. A. Creighton, C. G. Blatchford, M. G. Albrecht, J. Chem. Soc. Faraday Trans. II 1979, 75, 790.

[16] M. J. Frisch, G. W. Trucks, H. B. Schlegel, G. E. Scuseria, M. A. Robb, J. R. Cheeseman, J. A. Montgomery Jr., T. Vreven, K. N. Kudin, J. C. Burant, J. M. Millam, S. S. lyengar, J. Tomasi, V. Barone, B. Mennucci, M. Cossi, G. Scalmani, N. Rega, G. A. Petersson, H. Nakatsuji, M. Hada, M. Ehara, K. Toyota, R. Fukuda, J. Hasegawa, M. Ishida, V. Nakajima, Y. Honda, O. Kitao, H. Nakai, M. Klene, X. Li, J. E. Knox, H. P. Hratchian, J. B. Cross, C. Adamo, J. Jaramillo, R. Gomperts, R. E. Stratmann, O. Yazyev, A. J. Austin, R. Cammi, C. Pomelli, J. W. Ochterski, P. Y. Ayala, K. Morokuma, G. A. Voth, P. Salvador, J. J. Dannenberg, V. G. Zakrzewski, S. Dapprich, A. D. Daniels, M. C. Strain, O. Farkas, D. K. Malick, A. D. Rabuck, K. Raghavachari, J. B. Foresman, J. V. Ortiz, Q. Cui, A. G. Baboul, S. Clifford, J. Cioslowski, B. B. Stefanov, G. Liu, A. Liashenko, P. Piskorz, I. Komaromi, R. L. Martin, D. J. Fox, T. Keith, M. A. Al-Laham, C. Y. Peng, A. Nanayakkara, M. Challacombe, P. M. W. Gill, B. Johnson, W. Chen, M. W. Wong, C. Gonzalez, J. A. Pople, Gaussian 03 (Revision B.04), Gaussian, Inc, Pittsburgh, PA, 2003.

[17] C. Lee, W. Yang, R. G. Parr, Phys. Rev. B 1988, 37, 785.

[18] B. Miehlich, A. Savin, H. Stoll, H. Preuss, Chem. Phys. Lett. 1989, 157, 200.

[19] A. J. Becke, Phys. Rev. A 1988, 38, 3098.

[20] A. J. Becke, J. Chem. Phys. 1993, 98, 5648.

[21] P. J. Hay, W. R. Wadt, J. Chem. Phys. 1985, 82, 270.

[22] R. B. Grant, R. M. Lambert, J. Catal. 1985, 92, 364.

[23] Q. Tian, Y. Fang, Y. Sha, Molecules 2008, 13, 948.

[24] M. Pagannone, B. Fornari, G. Mattei, Spectrochim. Acta 1987, 43A, 621.

[25] Y. J. Kwon, D. H. Son, S. J. Ahn, M. S. Kim, K. Kim, J. Phys. Chem. 1994, 98, 8481.

[26] S. H. Choi, H. G. Park, Appl. Surf. Sci. 2005, 243, 76.

[27] X. Zhao, Y. Fang, J. Mol. Struct. 2006, 789, 157.

[28] J. L. Castro, M. R. Lopez-Ramirez, J. F. Arenas, J. Soto, J. C. Otero, Langmuir 2012, 28, 8926.

\section{Supporting information}

Additional supporting information may be found in the online version of this article at the publisher's web site. 